\title{
Dinámicas para las instituciones de educación superior del siglo XXI implementadas en el Bachillerato en la Enseñanza del Inglés de la Universidad Nacional de Costa Rica
}

\author{
Damaris Cordero Badilla \\ Escuela de Literatura y Ciencias del Lenguaje \\ Universidad Nacional \\ Olga Chaves Carballo \\ Escuela de Literatura y Ciencias del Lenguaje \\ Universidad Nacional
}

\begin{abstract}
Resumen
El objetivo de esta investigación exploratoria es conocer e indagar sobre la implementación de las recomendaciones dadas desde la primera Conferencia Mundial de Educación Superior, otras conferencias regionales (Cartagena de Indias, Macao, Dakar, Nueva Delhi, Bucarest y El Cairo), y las Conferencias Mundiales de Educación Superior 2009 y 2012 en el Bachillerato en la Enseñanza del Inglés (BEI), carrera acreditada de la Escuela de Literatura y Ciencias del Lenguaje (ELCL) de la Universidad Nacional (UNA). Se diseñó un cuestionario y se aplicó a un grupo de 13 estudiantes del BEI, 24 docentes del Área de Inglés y al director de la ELCL, además de una entrevista a la Rectora de la UNA realizada en los meses de abril y mayo de 2014. Los resultados revelan que el BEI estimula el pensamiento crítico, la creatividad, y las competencias profesionales que requieren los estudiantes y los docentes. Sin embargo, se requiere más investigación en los cursos. La Rectora indica que las recomendaciones de las conferencias internacionales seleccionadas para este estudio están aplicándose en la UNA a partir de la puesta en marcha de diferentes programas, proyectos y comisiones, así como en el modelo pedagógico.
\end{abstract}

Palabras claves: Conferencia Mundial sobre la Educación Superior, calidad, investigación, ética, modernización académica

\begin{abstract}
The aim of this exploratory research is to know and investigate the implementation of the recommendations made since the first World Conference on Higher Education, other regional conferences (Cartagena de Indias, Macau, Dakar, New Delhi, Bucharest and Cairo), and the World Conferences on
\end{abstract}


Higher Education 2009 and 2012 in the Bachelor of English Teaching (BEI), an accredited program, from the School of Literature and Language Sciences (ELCL) at Universidad Nacional. A questionnaire was designed and applied to a group of 13 students of the BEI, 24 English professors from the English department, the director of the ELCL and an interview with the President of the University held in the months of April and May, 2014. The results revealed that the BEI encourages critical thinking, creativity, and professional competences needed by the students and professors. However, more research is required in the courses. The President indicated that the recommendations of the International Conferences selected for this study are applied by UNA through the implementation of various programs, projects, committees, and the pedagogical model.

Key words: World Conference on Higher Education, quality, research, ethics, academic modernizing

\section{Introducción}

L as instituciones de educación superior (IES) cumplen una función importante en el desarrollo social y económico mundial, en la creación del conocimiento, en la formación de profesionales basada en las competencias y en la construcción de una nueva sociedad. De esta manera, para que estas organizaciones educativas cumplan sus fines deben promover la transformación y la renovación y poner en práctica una serie de recomendaciones que se vienen dando desde el año 1918 con la Reforma de Córdoba, en la Declaración de la Conferencia Mundial de Educación Superior 1998, en seis conferencias regionales (Cartagena de Indias, Macau, Dakar, Nueva Delhi, Bucarest y Cairo), y en los debates y resultados de la Conferencia Mundial "La nueva dinámica de la Educación Superior y de la Investigación para el Cambio Social y el Desarrollo", celebrada por la UNESCO en París, en el año 2009. Entre las recomendaciones mencionadas están: la igualdad de acceso, el estímulo del pensamiento crítico y la creatividad en los estudiantes y en los docentes, educar, formar y realizar investigación, ofrecer una mayor calidad en los procesos de enseñanza y aprendizaje, la modernización académica, la pertenencia y un cambio en el papel desempeñado ante la sociedad. Estos puntos también están considerados en la gestión de calidad de una carrera universitaria acreditada por el Sistema Nacional de Acreditación de la Educación Superior (SINAES) en Costa Rica.

La presente ponencia aborda las conclusiones de dichas conferencias, las acciones y los resultados que ha logrado la comunidad internacional para el cumplimiento de algunas de las misiones adquiridas por los estados subscritos. Con estos elementos, el objetivo de esta investigación exploratoria es conocer e investigar sobre la implementación de las recomendaciones antes mencionadas en el BEI, carrera acreditada, de la ELCL, con el fin de mantener las mejoras y los cambios pertinentes que conducen al cumplimiento de estándares, directrices, procedimientos de calidad y mejores 
prácticas universitarias que responden a la ética de la Educación Superior. Los argumentos estarán sustentados con datos recopilados y analizados a partir de un cuestionario diseñado por las investigadoras, aplicado a un grupo de 13 estudiantes del BEI, 24 docentes del Área de Inglés y del Director de la ELCL, así como por datos extraídos de una entrevista que se le realizó a la Rectora de la UNA en los meses de abril y mayo del año 2014.

\section{Contextualización del tema}

En la actualidad, muchos países están debatiendo si sus sistemas de educación superior son verdaderamente aptos y si estos contribuyen a la formación que los estudiantes y la sociedad necesitan. En países en desarrollo como el nuestro, en donde los recursos son escasos, se debe priorizar en instituciones que están cumpliendo con los propósitos más significativos para el país. En la Costa Rica de hoy, donde la calidad en la educación superior es una prioridad clave, las autoridades de educación toman en cuenta el interés de las personas, la necesidad del país, los costos, la calidad de la carrera y las normas internacionales para distribuir los recursos.

La educación superior en Costa Rica es la columna vertebral de esta sociedad y es la calidad de la educación universitaria la que decide la eficacia de los recursos humanos en el país. La educación superior, como la vemos actualmente, es un sistema complejo que facilita la enseñanza, la investigación, la extensión y la cooperación.

Las nuevas dinámicas de la educación superior y de la investigación para el cambio social y el desarrollo, propo- nen la formación de individuos íntegros con pensamiento crítico y ciudadanía activa quienes respondan a los problemas mundiales con dimensiones sociales, económicas, científicas y culturales y contribuyan con acciones para el desarrollo sostenible, los derechos humanos y el bienestar común, entre otros. La educación tiene el fin de promover el diálogo, no como forma de debate o discusión, sino el diálogo holista, el cual propone estar a la escucha del otro para descubrir juntos alternativas o soluciones que den respuestas a los temas que atañan a la sociedad actual.

En estas conferencias mundiales se acordaron ciertas misiones y funciones que las instituciones de educación superior (IES) necesitan cumplir para poder continuar durante el siglo XXI, tales como: la ausencia de discriminación en el acceso a estas instituciones, la evaluación de su pertinencia en función de lo que la sociedad espera de ellas y lo que éstas hacen, la diversificación de los modelos de educación y los métodos y los criterios de acceso a los estudiantes, así como la formación rigurosa necesaria para que ellos sean competentes en el siglo XXI. Así mismo, se debe:

garantizar de buenas condiciones profesionales y financieras a los académicos, estimulando en ellos la excelencia en la investigación y la enseñanza; situar a los estudiantes y sus necesidades en el centro de sus actividades, y considerarlos parte importante del proceso de renovación de la educación superior, la cooperación con el mundo del trabajo, el análisis y la previsión de las necesidades de la sociedad, y la diversificación como medio de reforzar la igualdad de oportunidades (UNESCO,1998, p. 2-3). 
Se propone exhortar los nuevos roles de los académicos en un mundo tan cambiante y lleno de contradicciones. Dentro de estos se incluye desarrollar una consciencia hacia los retos de la docencia:

...el docente universitario debe centrarse y asumir retos, para transformar las universidades, en centro educativo con una educación abierta, flexibles y sensibles en torno a lo local, regional y nacional. Convertir universidades donde formen estudiantes competitivos en los diferentes campos laborales, que sea idóneo para la adquisición de habilidades en el trabajo y la investigación en la producción de conocimiento aplicables donde los programas se centren en la contextualización de los diferentes problemas sociales del país y que a su vez responda en preparar profesionales en los distintos campos laborales (León, 2008, s.p.).

De esta manera, provocamos reinventar la formación del profesorado, o sea, buscar el alma de la profesión, ya que como indica Montessori (1986, p. 26): "La educación debe contribuir a un movimiento de liberación universal, indicando el modo de defender y elevar a la humanidad". Por lo tanto, es un desafío ser académico en la actualidad y debemos saber cómo desarrollar nuestro arte y ciencia satisfactoriamente en cuanto desarrollemos las competencias de investigación, transdiciplinariedad, responsabilidad social, liderazgo e identificación con los problemas de la comunidad.

\section{Las universidades latinoameri- canas en el siglo XXI}

Las instituciones de educación superior latinoamericanas han sufri- do varias reformas universitarias en el marco de la globalización y de la propia dinámica de la educación superior en el área, lo cual trae grandes cambios para estas organizaciones, tales como la demanda por más calidad en los procesos de enseñanza y aprendizaje, la internalización, la introducción y el uso de las nuevas tecnologías de comunicación e información (TIC). Así como la puesta en marcha de las recomendaciones para las universidades estipuladas en las diferentes conferencias internacionales de educación superior que se han venido dando desde el año 1998, entre otros. Eventos importantes que obligan a las IES a adaptarse al contexto actual y a desarrollar nuevas modalidades pedagógicas, alianzas internacionales y las habilidades y destrezas en los estudiantes y docentes que requiere la sociedad actual.

Sobre todo si se toma en cuenta que la educación superior en América Latina y el Caribe es una de las promotoras del desarrollo económico y la transformación social por lo que está obligada a replantear las visiones, las misiones, las estructuras y los currículos para hacerlos cada vez más flexibles y de acuerdo con lo que las economías exigen de ella, para educar y formar de esta manera a los futuros profesionales que demanda el mercado laboral del siglo XXI (Rama, 2006).

A raíz de lo anterior se han venido dando transformaciones recientes en las IES de América Latina, tales como: reformas en sus sistemas de evaluación, acreditación, fiscalización y control, el diseño de nuevos marcos legales, la ampliación de cursos a distancia, la creación de sistemas de becas y créditos, la internalización de la educación, la creditización de los cursos y carreras, la creación de indicadores de 
gestión de las universidades públicas y las tendencias hacia la especialización disciplinaria, entre otros (Lanz, Fergusson y Marcussi, 2003).

\section{La educación superior en Costa Rica}

Según la Coalición Costarricense de Iniciativas de Desarrollo (CINDE) en su Informe de La Educación en Costa Rica (2012, p. 9), en el país existen actualmente 59 universidades, cinco de las cuales son públicas: la Universidad de Costa Rica (UCR), el Instituto Tecnológico (TEC), la Universidad Nacional de Costa Rica (UNA), la Universidad Estatal a Distancia (UNED), y la más reciente institución la Universidad Técnica Nacional (UTN), y el resto son de privadas. Todas estas universidades son supervisadas por el Consejo Superior de la Educación Superior (CONESUP) y gozan de independencia para el desempeño de sus funciones, plena capacidad jurídica para adquirir derechos y contraer obligaciones, y para tener una organización y gobierno propios (CONARE, 2004, p.6).

En este país, las universidades juegan un papel transcendental en el desarrollo ya que no solo forman el recurso humano que asume la conducción de las empresas del sector privado y las instituciones públicas, sino que son la base de la innovación científica y tecnológica. No obstante, de 1.165 programas académicos impartidos en conjunto por el total de instituciones de educación superior universitaria, las 68 carreras que a marzo del 2013 habían recibido acreditación representaban solo un 5,7\% (Programa Estado de la Nación: Programa Estado de la Educación, 2013, p. 198). Además, este informe muestra cómo la oferta académica es pertinente para los requerimientos actuales del mercado, en donde solo un $2 \%$ de los egresados de las universidades tienen problemas de empleo, a pesar de que la cultura de evaluación para el mejoramiento continuo de la calidad "avanza a pasos lentos" (Programa Estado de la Nación: Programa Estado de la Educación, 2013, p. 199).

\section{Metodología del estudio}

Esta investigación es exploratoria con el fin de conocer e investigar sobre cada una de las recomendaciones de la UNESCO para compararlas con la realidad actual de las instituciones de educación superior en Costa Rica y en especial en la ELCL de la UNA. Los argumentos estarán sustentados con información recopilada por medio de un cuestionario que se aplicó en abril y mayo de 2014 a un grupo de 13 estudiantes del BEI de cuarto nivel, 24 docentes del Área de Inglés de la ELCL, el Director de la ELCL y la Rectora. El procedimiento fue el siguiente: a los estudiantes se les aplicó el cuestionario en su aula; a los docentes se les aplicó en una reunión del área y se aclararon varias preguntas pertinentes en un lapso de 40 minutos. El director de la ELCL completó el cuestionario vía correo electrónico y a la Rectora se le entrevistó en su oficina al otorgarnos una audiencia la primera semana de junio.

Los cuestionarios fueron diseñados por las investigadoras (ver apéndice 1). Estos se componen de dos partes: 3 preguntas personales y 8 preguntas sobre la implementación de 
algunas de las recomendaciones de la UNESCO y de las conferencias internacionales para las instituciones de educación superior. Se utilizó una escala de Likert con opciones de mucho, poco, nada, y una última pregunta de escogencia múltiple de tipos de estrategias de modernización académica que implementa la ELCL con los docentes del BEI.

En cuanto a los resultados, el $93 \%$ de los estudiantes está de acuerdo con la calidad en los procesos de enseñanza y aprendizaje que se imparten en el BEI. Es importante destacar las explicaciones de los estudiantes al respecto.

\section{Tabla 1}

\section{La calidad en los procesos de enseñanza y aprendizaje que se imparten en el BEI}

- Los profesores son muy buenos en su especialidad.

- Es bueno para ser la Universidad Nacional.

- Hay cursos de mucha calidad pero otros no lo son.

- En general los profesores son muy buenos y de ellos depende nuestro aprendizaje.

- Sí, tienen profesores con alto conocimiento en el área y el idioma.

- Considero bueno el proceso.

- Los contenidos y plan de estudios de los cursos son buenos, sin embargo, varios profesores no están capacitados para hacerlo.

- La calidad es buena por parte de casi todos los miembros de la ELCL.

- La calidad de la enseñanza es muy buena y siempre se busca lo mejor para el estudiante.

Fuente: Encuesta aplicada a estudiantes del BEI, UNA, en abril de 2014

\section{Cuadro 1 \\ Número de estudiantes y su evaluación al estímulo del pensamiento crítico}

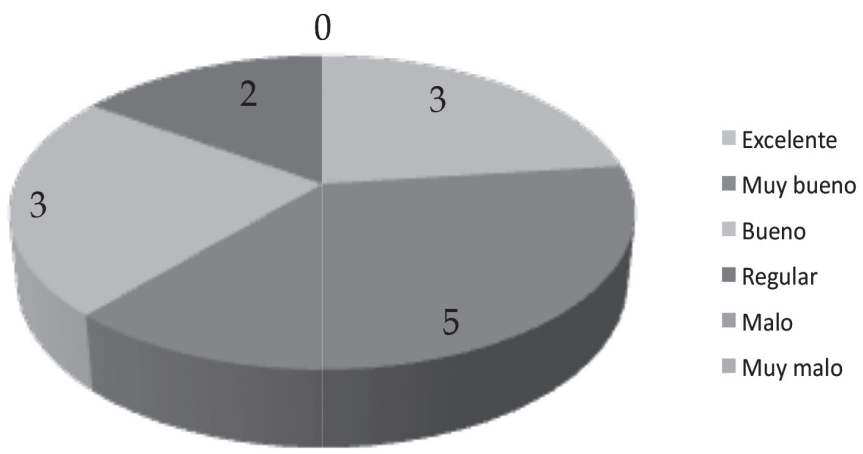

Igualmente, la evaluación al estímulo del pensamiento crítico y al estímulo de la creatividad por parte de profesores del BEI es muy buena de acuerdo con el 85\% de los estudiantes.
En cuanto a la forma para que se realicen investigaciones en cursos del BEI, 8 de los estudiantes entrevistados opinan que sí se estimula la investigación, pero 5 de estos dicen que no se estimula. 
A continuación se exponen las explicaciones de los estudiantes al referirse a la forma en que se realiza la investigación en los cursos del plan de estudio.

Por otra parte, los estudiantes concuerdan en que sí existe moderni- zación académica reciente. Además, la mayoría (10 de los 13) opinan que sí se les proporcionan las competencias profesionales que requieren para enfrentar el papel que deben asumir en la sociedad.

Tabla 2

Se le educa/forma para que realice investigaciones en cursos del BEI

- Aunque no nos enseñan investigación, es decir, nos mandan a investigar pero no nos enseñan el proceso formal.

- Por lo general en todos los cursos hay proyectos de investigación

- Sí, los cursos se basan en investigación.

- Muchos cursos tienen como proyecto realizar investigaciones.

- El uso de investigación se imparte hasta 4to año y es muy rápido, demandante y exagerado.

- Hasta el último año se enfocan en investigación, cuando uno está presionado con la práctica.

- No es visto en gran manera en gran importancia.

- Falta reforzarlo mucho.

- No se indica cómo deben llevarse a cabo, sino hasta cuando la carrera está por terminar.

- Sin embargo, faltan muchas bases en la investigación, especialmente en los cursos de primer y segundo años.

- La mayoría de los cursos incluyen investigación, sin embargo, las indicaciones que nos dan muchas veces nos limitan mucho e imponen barreras en cuanto a creatividad.

- Sí, pero son cursos muy confusos y en su mayoría no se explica y se entiende bien al estudiante.

- Todos, o casi todos los cursos trabajan en base a trabajos de investigación.

Fuente: Encuesta aplicada a estudiantes del BEI, UNA, en abril de 2014

Tabla 3

¿Se le proporcionan las competencias profesionales que requiere para enfrentar papel que debe asumir en sociedad?

- Se deben realizar proyectos en colegios.

- En cierta parte, sí, aunque considero que no como se debería.

- Hacen falta más cursos pedagógicos y sociales.

- De parte de la Escuela más bien se enseña más de lo necesario y está bien, pero de la parte pedagógica no.

- Proporcionan las herramientas para competir profesionalmente, pero personalmente siento que hace falta trabajar con las herramientas pedagógicas.

- ¿Qué hace un profesor con tener mucho conocimiento del idioma si no sabe cómo enfrentar las situaciones en la clase?

Fuente: Encuesta aplicada a estudiantes del BEI, UNA, en abril de 2014 
Por otro lado, el 92\% (23) de los académicos encuestados indican que sí estimulan el pensamiento crítico. Además, un 83.33\% (20 de los docentes) educa a los estudiantes para que realicen investigación en los cursos. No obstante, los estudiantes no valoran estas tareas como investigaciones, ya que opinan que se les debe estimular más para realizar investigaciones en todos los cursos del plan de estudio.

Sin embargo, todavía existe la necesidad de que a los docentes se les estimule el pensamiento crítico, la creatividad, la investigación y la capacitación por parte de la ELCL, ya que en la pregunta que se les incluyó en el cuestionario: "se les estimula para que realicen investigación en el campo de estudio de la ELCL", solo un 58.3\% (14) de ellos indican que "mucho" y el resto que "poco o nada".

Los docentes del BEI están muy satisfechos con los tipos de estrategias de modernización académica.

\section{Tabla 4}

\section{Comentarios acerca de tipos de estrategias de modernización académica}

\begin{tabular}{|c|c|}
\hline Comentarios & $\begin{array}{l}\text { Número de } \\
\text { docentes }\end{array}$ \\
\hline $\begin{array}{l}\text { A. Propicia la participación de los docentes y estudiantes en las } \\
\text { funciones básicas de la universidad. }\end{array}$ & 19 \\
\hline B. Fomento de la interdisciplinidad en el trabajo en equipo. & 19 \\
\hline C. Establece procesos de mejoramiento continuo. & 16 \\
\hline D. Oferta de programas de capacitación en diferentes áreas. & 16 \\
\hline $\begin{array}{l}\text { E. Promoción de la cultura fundamentada en principios de perte- } \\
\text { nencia, equidad y bienestar. }\end{array}$ & 15 \\
\hline F. Flexibilidad curricular. & 15 \\
\hline $\begin{array}{l}\text { G. Estímulo del manejo de diferentes lenguas extranjeras y dife- } \\
\text { rentes medios informáticos. }\end{array}$ & 13 \\
\hline
\end{tabular}

Fuente: Encuesta aplicada a académicos del BEI, UNA, en abril de 2014

De la misma manera, el Director de la ELCL confirma que el programa de estudios del BEI es accesible a todo aquel estudiante que finaliza satisfactoriamente su bachillerato en educación media y ha cumplido con todo el proceso de admisión de la Universidad Nacional. Además, comenta que en esta Escuela el pensamiento crítico y la creatividad se desarrollan en los estudiantes en todos los cursos. Considera también que varios de los cursos del BEI tienen un componente de investigación que se debe desarrollar. En cuanto a las capacitaciones a los docentes del BEI, dice que se cuenta con dos fondos: uno que permite contar con un especialista por año que pueda capacitarlos, y otro en diferentes áreas de desarrollo de la Escuela. 
Finalmente, la Rectora nos informa que todas las recomendaciones de las conferencias internacionales seleccionadas para este estudio están aplicándose en la UNA con la puesta en marcha de diferentes programas, proyectos y comisiones, y en el modelo pedagógico de la universidad. Sin embargo, considera que todavía en las diferentes escuelas se necesita flexibilizar más los programas de estudio para que puedan estar acordes con las recomendaciones planteadas en este estudio, y sugiere además la lectura del Informe de Gestión de la Rectoría del año 2012, en donde se detalla la forma en que esta institución de educación superior las ha implementado.

Como conclusión de este apartado sobre los resultados, podemos decir que es grato encontrar -según las respuestas dadas por la Rectora, el Director de la ELCL, los docentes y los estudiantes - que en la Universidad Nacional se está implementando la mayoría de las dinámicas para siglo XXI. Por otro lado, son pocas las dinámicas en las que se necesita trabajar para cumplir con las recomendaciones presentadas por los especialistas que asistieron a las Conferencias Mundiales para la Educación Superior.

\section{Conclusiones y recomendaciones}

Como conclusión y según la información recopilada, se puede decir que la UNA sí ha acogido y pone en práctica las recomendaciones que aquí se detallan, lo que la convierte en una institución de educación superior que se transforma y renueva para estar acorde con lo que este siglo XXI demanda de ella. En esta institución de educación superior:
- Existe igualdad de acceso para los estudiantes, sin importar su raza, religión, sexo u otra característica personal que los distinga.

- Se les estimula el pensamiento crítico a los estudiantes por parte de los profesores de la ELCL.

- Algunos de los profesores consideran que ellos necesitan estimular más el pensamiento crítico por parte de la ELCL.

- Los profesores estimulan la creatividad de los estudiantes en los cursos que imparten.

- Algunos de los profesores necesitan más estímulo a su creatividad por parte de la ELCL.

- Los profesores realizan investigaciones en su campo de estudio en la ELCL y, por su parte, educan y forman a sus estudiantes para que también la realicen.

Igualmente, el estudio revela que en algunas áreas, como la investigación y las capacitaciones, se debe continuar impulsando mejoras fundamentales para incorporar estas recomendaciones exitosamente y de esta manera estar al nivel de lo que exige este siglo XXI de las instituciones de educación superior.

En cuanto a la formación para realizar investigaciones en cursos del BEI, este es un aspecto que se debe mejorar en el programa, considerando que uno de los requisitos de una carrera acreditada es que las líneas de investigación de la carrera pertenezcan al plan de estudio. Igualmente, se ha instado y capacitado al personal académico del Área de Inglés para que incorporen tareas y proyectos de investigación en sus programas de curso. Dentro de los programas de curso, existen directrices para que el académico anote en 
la evaluación con detalle, las distintas tareas y proyectos que incluyen investigación. Además, en la bibliografía de los programas de los cursos se incorporan investigaciones realizadas por los académicos del Área de Inglés y de la División de Educología para promover la investigación en la academia.

\section{Bibliografía}

Águila, V. (2004). El Concepto Calidad en la Educación Universitaria: Clave para el Logro de la Competitividad Institucional. Recuperado de:http://www.rieoei.org/rie35.htm.

ANUIES (1998). La Educación Superior en el Siglo XXI: Una propuesta de la ANUIES. Recuperado de: http://publicaciones.anuies.mx/ revista/113/5/2/es/la-educacion-superior-en-el-siglo-xxi-lineas-estrategicas-de.

Arellano, J. (2002). Competitividad internacional y educación en los países de América Latina y el Caribe. The Latin American Competitiveness Report 2001-2002, World Economic Forum, Center for International Development, Harvard University, Oxford University Press.

Beneitone, P., Esquetini, C. y González, J. (2007) Reflexiones y perspectivas de la Educación Superior en América Latina: Informe Final - Proyecto Tuning - América Latina 2004-2007. España: Universidad de Deusto.

Brunner, J. (2001). La universidad en el siglo XXI. Guatemala: MINEDUC. (Cuadernos Pedagógicos, No. 15).

Coalición Costarricense de Iniciativas de Desarrollo (CINDE) (2012).
Educación en Costa Rica. Recuperado de: www.cinde.org/.../079_ Educacion\%20en\%20Costa\%20 Rica.pdf.

Comboni, S. y Juárez, J. (1997). La educación superior en América Latina: perspectivas frente al siglo $X X I$. Recuperado de: www.redalyc. org/pdf/267/26700902.pdf.

Consejo Nacional de Rectores (CONARE) (2013). Estado de la educación costarricense: Programa Estado de la Nación. Recuperado de: http://www.estadonacion.or.cr/.

Consejo Nacional de Rectores (CONARE) (2004). Oficina de Planificación de la Educación Superior. La Educación Superior Universitaria en Costa Rica. Recuperado de: http://tuning.unideusto.org/tuningal/images/stories/presentaciones/ costa_rica.pdf.

Cortez, J. (2012). Foro II: Tendencias y Retos de la Educación Superior en el Mundo. Las Conferencias Mundiales sobre la Educación Superior: objetivos y algunos avances. Recuperado de http://sincronia.cucsh. udg.mx/lozanocampos04.htm.

Enríquez, J. (2005) Educación superior: tendencias y desafíos. Recuperado de: www.ffa.sld.cu/post-materialesdiplomadopedagogia-doc1.pdf.

Gazzola, A. y Didriksson, A. (2008). Tendencias de la Educación Superior en América Latina y el Caribe. Recuperado de: http://www.unesco. org.ve/index.php?option=com_cont ent\&view=article\&id=2\&Itemid=4 $08 ?=\mathrm{es}$.

Lanz, R. Fergusson, A. y Marcussi, A. (2003). Procesos de Reforma de la Educación Superior en América Latina. Informe Sobre la Educación Superior en América Latina 
y el Caribe: 2000-2005. La Metamorfosis de la Educación Superior. Instituto Internacional de la UNESCO para la Educación Superior en América Latina y el Caribe (IESALC). ISBN -980-6556-19-4. Recuperado de: http://www.redetis.iipe.unesco.org/publicaciones/ informe-sobre-la-educacion-superior-en-america-latina-y-el-caribe-2000-2005/\#.VAs-s8KwIoB.

León, C. (2008). El docente universitario: retos, perspectivas, concepción. San Cristóbal. En línea. Recuperado de http://www.monografias.com/ trabajos 75/docente-universitarioretos-perspectivas-concepcion/docente-universitario-retos-perspectivas-concepcion.shtml\#eldocentea. Lozano, M. y Campos, H. (2004). El papel del docente de educación superior en la sociedad globalizada. SINCRONIA: A Journal for the Humanities and Social Sciences. Department of Literature and Department of Philosophy.University of Guadalajara.Winter 2011. Recuperado de: http://sincronia.cucsh. udg.mx/lozanocampos04.htm.

Montessori, M. (1986). Formación del hombre. México: Editorial Diana.

Moreno-Brid, J. y Ruiz-Nápoles, P. (2009). La educación Superior y el desarrollo económico en América Latina. México, D.F.: CEPAL, Naciones Unidas. Recuperado de: http://www.eclac.org/publicaciones/ $\mathrm{xml} / 5 / 35095 /$ Serie_106.pdf.

Programa Estado de la Nación: Programa Estado de la Educación (2013). Capítulo IV. La Evaluación de la Educación Superior. Hallazgos Relevantes. Recuperado de: http://www. estadonacion.or.cr/index.php/estadoeducacion/educacion-informe-ultimo.
Rama, C. (2006). La Tercera Reforma de la Educación Superior en América Latina y el Caribe. Fondo de Cultura Económica, Buenos Aires. Edición: 1, 240 pp, Publisher: Fondo de Cultura Económica, Editor: FCE, Buenos Aires, ISBN: 950557-6773. Recuperado de: http:// www.google.com/url?sa $=$ t\&rct $=$ j $\& \mathrm{q}=\&$ esrc $=\mathrm{s} \&$ source $=$ web $\& \mathrm{~cd}=3$ $\&$ ved $=0 \mathrm{CC} 4 \mathrm{QFjAC} \& \mathrm{url}=\mathrm{http} \% 3$ A $\% 2 \mathrm{~F} \% 2 \mathrm{Fwww}$.epuss.edu.pe $\% 2 \mathrm{~F}$ Documentos\%2FDEAC\%2FGest ion $\% 2$ FLatercerareforma-masificacionyreforma.pdf\&ei=7B0JVM KBJYa2ogTI1YCADQ\&usg=AFQ jCNE22_eiHpMkHEAAmsZ3FTs 6xr99sQ\&sig2=0NhD-UUgqgbJ t3GyNbpIw\&bvm=bv.74649129,d. cWc.

Rama, C. (2007). Tendencias de la educación superior en el siglo XXI. Recuperado de: http://noticias.universia.edu.pe/vida-universitaria/ noticia/2007/06/19/747349/tendencias-educacion-superior-siglo-xxi. html.

Rama, C. (2010). La Tercera Reforma de la Educación Superior en América Latina. Editorial: FONDO DE CULTURA ECONOMICA DE ESPAÑA, S.L.

Ruiz, A. (2000). La Educación Superior en Costa Rica: Tendencias y Retos en un nuevo escenario histórico. San José: Editorial de la Universidad de Costa Rica.

Ruiz, A. (2001). El papel de la Universidad: una radiografía de nuestra época y las tendencias en la educación superior. San José: Editorial de la Universidad de Costa Rica.

Segura, O. (2010). Universidad y desarrollo: desafíos en el siglo XXI. Heredia: CINPE, 2010. 
Tünnermann, C. y Fernández, F. (1997). La educación superior frente al cambio. San José, C.R. : EDUCA. CSUCA. (Colección 50 aniversario CSUCA 1948-1998; No. 1).

Tünnermann, C. (1998). La transformación de la educación superior: retos y perspectivas. Heredia: EUNA. Tünnermann, C. (2008). La Calidad de la Educación Superior y su Acreditación: la experiencia Centroamericana. Recuperado de: www.scielo. br/pdf/aval/v13n2/05.pdf.

Tyler, L. y Bernasconi, A. (1999). "Evaluación de la Educación Superior en América Latina-tres Órdenes de Magnitud". Harvard Institute for Internacional Development.
Harvard University. Recuperado de: www.cid.harvard.edu/hiid/700. pdf.

UNESCO. (1998). Conferencia Mundial sobre la Educación Superior: La educación superior en el siglo XXI. Visión y acción UNESCO, París, 5 -9 de octubre de 1998. Tomo 1. Informe Final. Recuperado de: http:// www.unesco.org/education/educprog/wche/declaration_spa.htm.

UNESCO. (2006). Informe sobre la Educación Superior en América Latina y el Caribe. 2000-2005: La metamorfosis de la educación superior. Recuperado de: http://www.redetis.org.ar/node. php?id=137\&elementid $=74$.

\title{
ANEXO
}

UNIVERSIDAD NACIONAL

Escuela de Literatura y Ciencias del Lenguaje

Bachillerato en la Enseñanza del Inglés

\section{Cuestionario Percepción de los Estudiantes \\ Cambios realizados para la transformación y renovación en Gestión de Calidad del BEl}

\begin{abstract}
Las instituciones de educación superior; como la Universidad Nacional (UNA); para poder operar en este Siglo XXI deben promover su transformación y renovación, pero para que esto suceda deben poner en práctica varias recomendaciones dictadas por organismos internacionales como la UNESCO y en conferencias internacionales sobre las Educación Superior. Es con esto en mente que el objetivo de este sondeo exploratorio es el de conocer e investigar la realidad que se vive en la Escuela de Literatura y Ciencias del Lenguaje (ELCL) con respecto a los cambios para Gestión de Calidad que se han venido dando, en el BEI y las estrategias utilizadas para la puesta en marcha e implementación de estas recomendaciones. Respetuosamente se le solicita su colaboración para conocer su percepción sobre los cambios realizados.
\end{abstract}

Sexo: M ( ) H ( )

Edad

Estudiante 
1. ¿Cómo evalúa usted el estímulo al pensamiento crítico en los estudiantes por parte de los profesores del BEI?

$\begin{array}{ll}\text { Excelente ( ) } & \text { Muy bueno ( ) } \\ \text { Bueno () } & \text { Regular ( ) } \\ \text { Malo () } & \text { Muy malo ( ) }\end{array}$

2. ¿Cómo evalúa usted el estímulo a la creatividad de los estudiantes por parte de los profesores del BEI?

$$
\begin{array}{ll}
\text { Excelente ( ) } & \text { Muy bueno ( ) } \\
\text { Bueno () } & \text { Regular ( ) } \\
\text { Malo () } & \text { Muy malo ( ) }
\end{array}
$$

3. ¿Considera usted que en la ELCL se estimula la igualdad de acceso al BEI a todo tipo de estudiante?

Sí ( ) No ( )

Explique:

4. ¿Se le educa/forma a usted para que realice investigaciones en los cursos del BEI?

Sí ( ) No ( )

Explique:
5. ¿Ofrece la ELCL calidad en los procesos de enseñanza y aprendizaje que se imparte en el BEI?

Sí ( ) No ( )

Explique:

6. ¿Se ha realizado en la ELCL alguna modernización académica recientemente?

Sí ( ) No ( )

Explique:

7. ¿Considera usted que en el BEI le proporcionan las competencias profesionales que requiere para enfrentar el papel que debe asumir en sociedad?

Sí ( ) No ( )

Explique:

8 ¿Desearía agregar un último comentario para la mejora en la Gestión de Calidad del BEI?

Sí ( ) No ( )

Explique:

¡Muchas gracias! 
